\title{
Avaliação do perfil nictemeral de excreção de indicadores internos e de óxido crômico em ensaios de digestão com ruminantes
}

\author{
Emanoel Elzo Leal de Barros ${ }^{1}$, Carlos Augusto de Alencar Fontes ${ }^{2}$, Edenio Detmann ${ }^{3}$, \\ Ricardo Augusto Mendonça Vieira ${ }^{2}$, Lara Toledo Henriques ${ }^{1}$, Enilson Geraldo Ribeiro ${ }^{1}$
}

\footnotetext{
${ }^{1}$ Doutor em Produção Animal.

2 LZNA/CCTA/UENFBolsista do CNPq.

${ }^{3}$ DZO/UFV. Bolsista do CNPq.
}

RESUMO - Objetivou-se com este trabalho avaliar a dinâmica de excreção fecal dos indicadores internos matéria seca indigestível (MSi), fibra em detergente neutro indigestível (FDNi), fibra em detergente ácido indigestível (FDAi) e do indicador externo óxido crômico $\left(\mathrm{Cr}_{2} \mathrm{O}_{3}\right)$. Foram utilizados cinco bovinos mestiços Holandês $\times$ Zebu, com peso médio de $240 \mathrm{~kg}$, em três períodos experimentais de 12 dias (os sete primeiros destinados à adaptação dos animais). Antes do início do período experimental, os animais passaram por um período de adaptação à dieta e ao manejo, com duração dez dias, no qual foram mantidos em baias individuais e alimentados à vontade com silagem de sorgo. $\mathrm{O}_{2} \mathrm{O}_{3}$ foi fornecido diariamente em duas porções de $5 \mathrm{~g}$, às $8 \mathrm{~h}$ e $17 \mathrm{~h}$, durante todo o período experimental. Procedeu-se à caracterização do padrão diurno de excreção fecal dos indicadores, por meio do algoritmo de Gauss-Newton, em um modelo em série de Fourier. A avaliação descritiva das funções ajustadas evidenciou que a amplitude total dos ciclos foi menor para FDAi que para outros indicadores, com valores de 4,71; 1,$83 ; 2,42$ e $1,74 \%$ para $\mathrm{Cr}_{2} \mathrm{O}_{3}, \mathrm{MSi}$, FDNi e FDAi, respectivamente, em relação à concentração média estimada para cada um desses indicadores. Por sua vez, o período fundamental de excreção dos indicadores em estudo foi significativo e evidenciou duração do ciclo de 3,$25 ; 3,47 ; 3,46$ e 3,74 horas para o $\mathrm{Cr}_{2} \mathrm{O}_{3}$, MSi, FDNi e FDAi, respectivamente. A concentração média fecal dos indicadores pode ser obtida por meio de duas coletas diárias de fezes, mantendo-se o espaço de tempo entre duas coletas consecutivas de $3,9,15$ ou 21 horas.

Palavras-chave: excreção fecal, fibra em detergente ácido indigestível, fibra em detergente neutro indigestível, matéria seca indigestível

\section{Evaluation of excretion patterns of internal markers and chromium oxide in digestion assays with ruminants}

\begin{abstract}
The goal of the present study was to evaluate the dynamics of fecal excretion of the following digestion markers: indigestible dry matter (DMi), indigestible neutral detergent fiber (NDFi), and indigestible acid detergent fiber (ADFi) as internal markers and the external marker chromic oxide $\left(\mathrm{Cr}_{2} \mathrm{O}_{3}\right)$. Five Holstein-zebu steers with $240 \mathrm{~kg}$ average weight were used in three 12-day experimental periods, being the first seven days for animal adaptation. Previously to the beginning of the experimental period, the animals were submitted to a 10-day period of adaptation to facilities and experimental management. They were fed ad libitum with sorghum silage and maintained in individual pens. $\operatorname{The} \mathrm{Cr}_{2} \mathrm{O}_{3}$ was offered twice daily at 08:00 and 17:00 h, $5 \mathrm{~g}$ each, during the whole experimental period. Then, the daily marker fecal excretion pattern was characterized by means of the Gauss-Newton algorithm to fit a Fourier series model. A descriptive graphic evaluation of the fitted function showed that the total amplitude of cycles was lower for ADFi than the other markers, with values of $4.71 \%$, $1.83 \%, 2.42 \%$ and $1.74 \%$ for $\mathrm{Cr}_{2} \mathrm{O}_{3}, \mathrm{DMi}, \mathrm{NDFi}$ and $\mathrm{ADFi}$, respectively, in relation to the estimated mean concentration of each. In turn, the fundamental period of the markers excretion patterns were significant showing cycle lengths of 3.25 , 3.47, 3.46 and 3.74 hours for $\mathrm{Cr}_{2} \mathrm{O}_{3}$, DMi, NDFi and ADFi, respectively. As a conclusion, the mean fecal concentration of all markers might be obtained through two daily samplings at intervals from each other of 3, 9, 15 or 21 hours.
\end{abstract}

Key Words: fecal excretion, indigestible acid detergent fiber, indigestible dry matter, indigestible neutral detergent fiber

\section{Introdução}

O êxito dos ensaios de digestão conduzidos com animais ruminantes depende da acuidade de medição de variáveis como o consumo alimentar, a produção fecal e a própria digestibilidade. No Brasil, este assunto apresenta relevância zootécnica em razão da maneira como os animais são criados, o que exige avaliação sistemática para o aperfei- 
çoamento de procedimentos para obtenção de estimativas que permitam realizar inferências válidas nessas circunstâncias.

Sem dúvida, a coleta total de fezes e os registros das quantidades de matéria alimentar oferecida e refugada pelos animais são as medidas diárias que melhor permitem estimar o balanço da matéria alimentar e os demais itens que, em conjunto, possibilitam encontrar o valor nutritivo dos alimentos. Todavia, sua aplicação traz várias restrições de mobilidade, mão-de-obra e acessibilidade ao que é necessário medir, que suscita estudos com indicadores para facilitar a obtenção das estimativas referentes aos ensaios de digestão (Owens \& Hanson, 1992; Faichney, 1993; Van Soest, 1994). Recomenda-se cautela nestes estudos pelo fato de as concentrações fecais dos indicadores sofrerem interferências de fatores que causam variações sistemáticas em seus padrões de excreção e viciam a estimação da produção diária de matéria fecal (Hopper et al., 1978; Oliveira et al., 1991; Owens \& Hanson, 1992).

A recuperação de frações indigestíveis do alimento é a base para os indicadores internos, que são utilizados convenientemente em estudos envolvendo estimativas de digestibilidade. Os indicadores internos comumente usados são a fibra em detergente neutro indigestível (FDNi), a fibra em detergente ácido indigestível (FDAi) e a matéria seca indigestível (MSi) (Penning \& Johnson, 1983; Cochran et al., 1986; Lippke et al., 1986; Van Soest, 1994; Titgemeyer, 1997; Detmann et al., 2001)

A base para a estimação da excreção de matéria seca fecal é o conhecimento da quantidade do indicador fornecido diariamente aos animais e a concentração desse indicador nas amostras fecais, coletadas em intervalos de tempo definidos durante o dia. Um aspecto deste procedimento que requer atenção especial é a taxa de excreção dos indicadores nas fezes ao longo de um período de 24 horas (Brisson et al., 1957; Hopper et al., 1978).

O óxido crômico tem sido historicamente empregado em virtude de seu baixo custo, da praticidade e por não violar a maior parte das pressuposições que definem um indicador ideal. O seu uso, combinado às frações indigestíveis da matéria alimentar, tem proporcionado resultados variáveis de estimativas associadas aos ensaios de digestão no Brasil (Oliveira et al., 1991; Piaggio et al., 1991; Berchielli et al., 2000; Detmann et al., 2001; Zeoula et al., 2002), o que justifica a realização de estudos que contribuam para a identificação de possíveis fatores interferentes.

As variações observadas podem violar a pressuposição de equilíbrio para a excreção média diária dos indicadores, o que não pode ser corrigido apenas com a amostragem sistemática em pontos acima e abaixo das inflexões do perfil, tampouco pela composição de amostras a partir de quantidades iguais extraídas das amostras de fezes colhidas pela manhã ou à tarde. A análise dos perfis de excreção, entretanto, pode auxiliar no entendimento de como os horários de coleta podem afetar as estimativas (Hopper et al., 1978).

Neste estudo, objetivou-se avaliar os perfis de excreção dos indicadores internos FDNi, FDAi, MSi e do óxido crômico $\left(\mathrm{Cr}_{2} \mathrm{O}_{3}\right)$ em um ensaio de digestão com bovinos.

\section{Material e Métodos}

Foram utilizados cinco bovinos Holandês $\times$ Zebu, machos não-castrados, com peso corporal médio de $240 \mathrm{~kg}$, alojados em baias individuais. A alimentação fornecida foi constituída exclusivamente por silagem de sorgo, fornecida à vontade, sempre às $7 \mathrm{~h} 30$, admitindo-se sobras de $10 \% \mathrm{da}$ quantidade do alimento fornecido. Amostras destas sobras e do alimento fornecido foram acondicionadas em sacos plásticos e conservadas congeladas para posteriores análises. Inicialmente, os animais foram mantidos por um período de adaptação à dieta e ao manejo, com duração de dez dias.

Foram administradas duas doses diárias de $\mathrm{Cr}_{2} \mathrm{O}_{3}$ contendo $5 \mathrm{~g}$, sempre às $8 \mathrm{~h}$ e às $17 \mathrm{~h}$, com o auxílio de uma sonda esofagiana (Hopper et al., 1978). O período de adaptação foi de sete dias, antes do início do período de coletas fecais, com o objetivo de se alcançar um platô de concentração desse indicador nas fezes. $\mathrm{O}$ fornecimento do $\mathrm{Cr}_{2} \mathrm{O}_{3}$ foi mantido até o final dos três períodos de coleta.

As amostras fecais foram coletadas em três períodos seqüenciais de seis dias, com intervalo de cinco dias entre períodos. Durante estes períodos, foram realizadas coletas totais e amostrais em todos os cinco animais, concomitantemente. As coletas totais foram realizadas durante o primeiro, terceiro e quinto dias de cada período de coleta durante 24 horas, contadas a partir das $7 \mathrm{~h}$. As fezes obtidas pela coleta total foram pesadas para determinação da produção fecal total e homogeneizadas, retirando-se alíquotas de $10 \%$, que foram acondicionadas em sacos plásticos e congeladas a $-20^{\circ} \mathrm{C}$ para posteriores análises.

Durante o segundo, o quarto e o sexto dias de cada período de coletas, realizaram-se coletas intermitentes de fezes, a partir das $7 \mathrm{~h}$, em intervalos de 1 hora e 30 minutos. As amostras foram acondicionadas e conservadas de forma similar às amostras obtidas por coleta total.

Posteriormente, as amostras de fezes foram descongeladas em temperatura ambiente, secas em estufa de ventila- 
ção forçada $\left(60^{\circ} \mathrm{C}\right.$ por 72 horas) (Silva \& Queiroz, 2002), processadas em moinho de facas com peneira de porosidade de $2 \mathrm{~mm}$ e acondicionadas em frascos de polietileno, devidamente identificados. $\mathrm{O}$ mesmo procedimento foi adotado para as amostras de alimento e sobras. Após o processamento físico, estas amostras e as amostras fecais resultantes das coletas totais foram compostas, proporcionalmente, com base no peso seco ao ar, por animal por período.

Após a estimação do teor de MS total (Silva \& Queiroz, 2002), alíquotas de $1,1 \mathrm{~g}$ das amostras de alimentos, sobras e fezes (coletas totais e amostrais) foram acondicionadas, em duplicata, em sacos de náilon de $6 \times 3,5 \mathrm{~cm}$, com porosidade de $50 \mu \mathrm{m}$, previamente pesados e identificados. Os sacos foram selados a quente e incubados durante 144 horas no rúmen de um bovino adulto alimentado com a mesma dieta fornecida durante os períodos de coleta. Posteriormente, foram retirados, lavados até o completo clareamento da água, levados a estufa de ventilação forçada a $60^{\circ} \mathrm{C}$ por 72 horas e submetidos à secagem em estufa a $105^{\circ} \mathrm{C}$ por 1 hora. Em seguida, foram retirados, esfriados em dessecador e pesados para determinação da concentração de MSi. Posteriormente, foram submetidos à fervura em detergente neutro (Goering \& Van Soest, 1970), por 1 hora, lavados com água quente e acetona, secos e pesados, pelo mesmo procedimento, para determinação da FDNi. Após a pesagem, foram novamente submetidos à fervura em detergente ácido (Goering \& Van Soest, 1970) adotando-se o mesmo procedimento utilizado na determinação da FDAi. A quantidade de detergente utilizada foi de $50 \mathrm{~mL}$ por saco.

Posteriormente, as amostras fecais obtidas por coletas totais e amostrais foram quantificadas quanto ao teor de cromo, com preparação de solução mineral segundo método descrito por Kimura \& Müller(1957). A avaliação quantitativa foi realizada por espectrofotometria de absorção atômica determinando-se ainda o teor de cromo no óxido crômico utilizado para quantificação da pureza do produto.

A partir das informações obtidas nas coletas de fezes, procedeu-se à descrição do perfil nictemeral de cada indicador, por meio de ajuste do modelo não-linear em série de Fourier, descrito por Hopper et al. (1978), aos perfis de excreção:

$$
\begin{gathered}
\mathrm{Y}=\mathrm{A}_{0}+\mathrm{A}_{1} \operatorname{sen}(\mathrm{ct})+\mathrm{A}_{2} \cos (\mathrm{ct})+\mathrm{A}_{3} \operatorname{sen}(2 \mathrm{ct})+\mathrm{A}_{4} \\
\cos (2 \mathrm{ct})+\mathrm{e}
\end{gathered}
$$

em que: $\mathrm{Y}=$ concentração fecal predita do indicador $(\mathrm{g} / \mathrm{g} \mathrm{MS}$ fecal) no tempo t; $\mathrm{A}_{0}=$ concentração média do indicador (g/g MS fecal); $\mathrm{c}=$ período fundamental ou comprimento do ciclo $(\mathrm{rad} / \mathrm{h}) ; \mathrm{t}=$ tempo em horas; $\mathrm{A}_{1}, \mathrm{~A}_{2}, \mathrm{~A}_{3}, \mathrm{~A}_{4}=$ parâmetros de escala sem significado biológico; e o termo e corresponde ao erro aleatório, suposto normal e independentemente distribuído, com média zero e variância $\sigma^{2}$.
O ajuste não-linear foi realizado por meio do procedimento iterativo de Gauss-Newton implementado no procedimento NLIN (SAS, 1993). Ressalta-se que o modelo foi ajustado sobre o conjunto de dados totais obtidos no experimento para cada indicador $(\mathrm{n}=761)$. Quando pertinente, estatísticas adicionais (erro-padrão assintótico e intervalo de confiança assintótico) foram associadas às estimativas dos parâmetros obtidos. Posteriormente ao ajuste, procedeu-se à estimação da duração do período fundamental de oscilação da concentração fecal do indicador:

$$
t_{c}=\frac{\pi}{c}
$$

em que: $t_{c}=$ duração do período fundamental de oscilação da concentração fecal do indicador (h); $\pi$ = base para a avaliação nictemeral (3,1416 rad); e c = período fundamental de oscilação da concentração fecal do indicador (rad/hora).

Em adição à avaliação do perfil excretório, procedeu-se à estimação da recuperação fecal de cada indicador a partir das equações:

$$
\begin{gathered}
R F=\left(\frac{E F R}{C I}\right) \times 100 ; \\
E F R=\frac{C F I}{C I} ;
\end{gathered}
$$

em que: $\mathrm{RF}=$ recuperação do indicador (\%); EFR = estimativa da excreção do indicador obtida a partir de sua concentração na amostra fecal obtida via coleta total de fezes (g/ dia); CFI = concentração do indicador na amostra fecal obtida via coleta total $(\mathrm{g} / \mathrm{g}) ; \mathrm{CI}=$ consumo ou dose diária do indicador (g/dia).

Sobre as estimativas de recuperação fecal dos indicadores construíram-se intervalos de confiança com $95 \%$ de probabilidade. Neste procedimento empregou-se estimativa da variação residual obtida por ANOVA a partir de um modelo contendo os efeitos de animal e período de coleta fecal.

\section{Resultados e Discussão}

Uma condição prévia para a estimação precisa da produção fecal é que a concentração do indicador utilizado seja constante ao longo do dia ou siga um padrão de excreção diurno definido (Brisson et al., 1957).

Os ritmos preditos (Figura 1) foram caracterizados como os do tipo estacionário, resultado da flutuação normal da concentração dos indicadores estudados nas fezes durante o período experimental.

As equações obtidas pelo ajuste não linear das concentrações dos indicadores cromo, matéria seca indigerível 

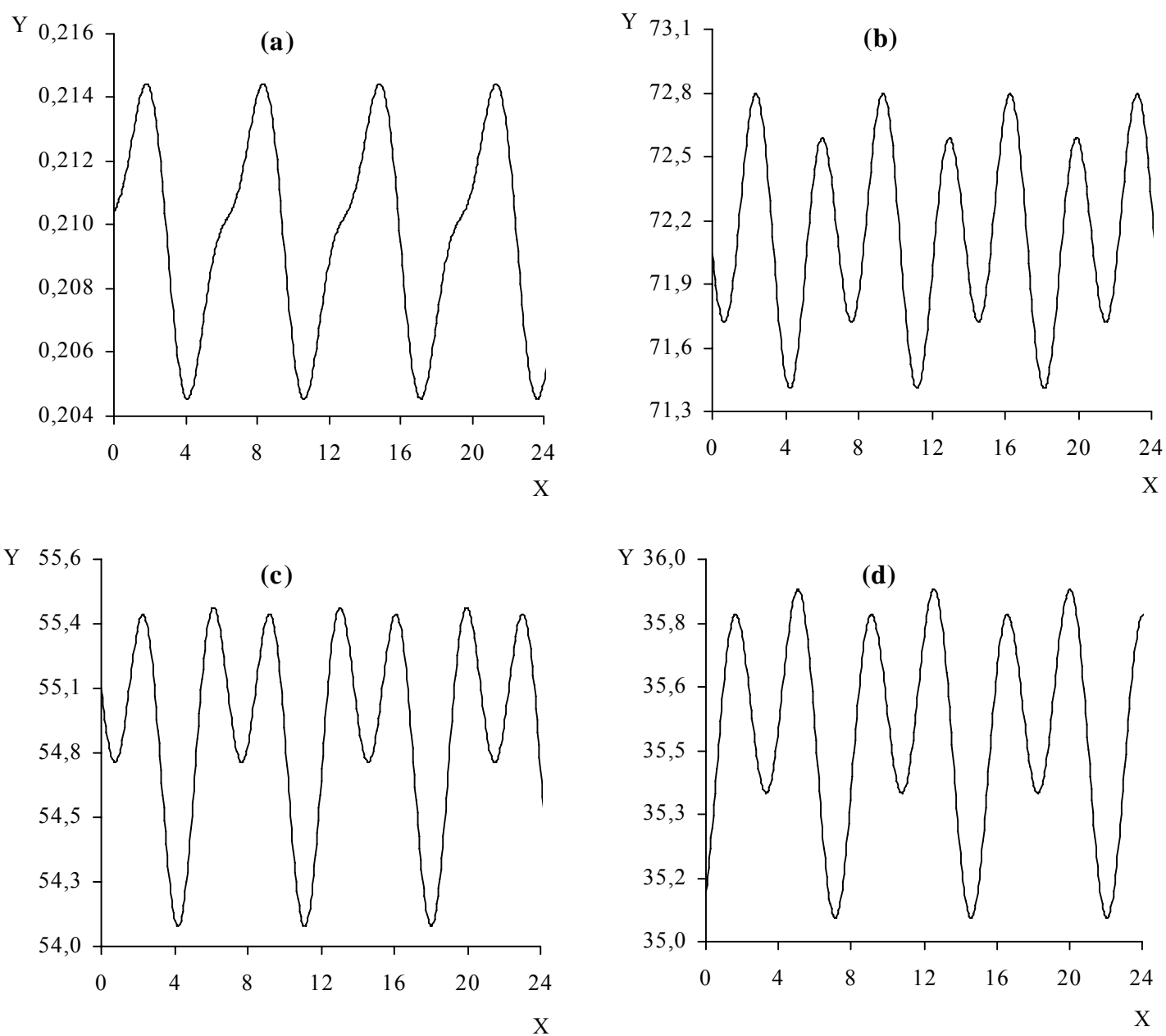

Figura 1 - Perfis nictemerais esperados para a concentração fecal (\%MS) dos indicadores (a) cromo, (b) matéria seca indigerível (Msi), (c) fibra em detergente neutro indigerível (FDNi), e (d) fibra em detergente ácido indigerível (FDAi), dispostas nas ordenadas (eixos $\mathrm{Y}$ ) em função do tempo de amostragem (eixos $\mathrm{X}$ ).

Figure 1 - Expected nicterohemeral fecal concentration profiles (\%DM) of the markers (a) cromium, (b) indigestible dry matter (DMi), (c) indigestible neutral detergent fiber (NDFi), and (d) indigestible acid detergent fiber (ADFi), plotted on the ordinates ( $Y$ axis) and as functions of sampling time ( $X$ axis).

(Msi), fibra em detergente neutro indigerível (FDNi) e fibra em detergente ácido indigerível (FDAi) em relação ao tempo de amostragem $(\mathrm{t})$ foram: cromo $=0,2097+$ $0,0039 \operatorname{sen}(0,9653 t)+0,0014 \cos (0,9653 t)-0,0015 \operatorname{sen}(1,9306 t)$ $-0,0007 \cos (1,9306 \mathrm{t}) ; \mathrm{Msi}=72,14+0,1725 \operatorname{sen}(0,9046 \mathrm{t})+$ $0,058 \cdot \cos (0,9046 \mathrm{t})-0,5215 \operatorname{sen}(1,8092 \mathrm{t})-0,1423 \cos (1,8092 \mathrm{t})$; $\mathrm{FDNi}=54,9+0,2037 \operatorname{sen}(0,9086 \mathrm{t})+0,2765 \cos (0,9086 \mathrm{t})-$ $0,4612 \operatorname{sen}(1,8172 t)-0,1031 \cdot \cos (1,8172 t) ; F D A i=35,53+$ $0,0206 \operatorname{sen}(0,84 t)-0,1586 \cos (0,84 t)+0,1930 \operatorname{sen}(1,68 t)-$ $0,2465 \cos (1,68 \mathrm{t})$.

Apesar de a escala adotada para construção dos gráficos evidenciar picos e vales nos perfis de excreção, a amplitude entre esses valores esperados foi relativamente pequena, com destaque para o teor de cromo nas fezes, que apresentou maior amplitude porcentual em relação aos indicadores internos (Tabela 1).
A excreção fecal mais estável para os indicadores internos é indicativo de que esses indicadores apresentaram fluxos de escape que menos violaram a pressuposição de equilíbrio para a excreção. $\mathrm{O}$ fornecimento de duas doses diárias para o $\mathrm{Cr}_{2} \mathrm{O}_{3}$, como esperado, proporcionou descontinuidade que pareceu violar a pressuposição acima. Todavia, pela amplitude observada, e se tomados os cuidados para uma amostragem representativa (Hopper et al., 1978), permite-se admitir que o emprego do óxido crômico possibilitaria a obtenção de estimativas válidas para a produção de matéria seca fecal (Oliveira et al., 1991; Detmann et al., 2001).

A duração do período fundamental não diferiu $(\mathrm{P}>0,05)$ entre os indicadores estudados (Tabela 2). As estimativas obtidas foram de 3,25, 3,47, 3,46 e 3,74 horas para o $\mathrm{Cr}_{2} \mathrm{O}_{3} \mathrm{e}$ os indicadores internos $\mathrm{MSi}$, FDNi e FDAi, respectivamente. 
Tabela 1 - Concentrações fecais médias $\left(A_{0}-\%\right)$, concentrações fecais máxima e mínima estimadas (\%) e amplitude total de oscilação para os indicadores óxido crômico, matéria seca indigestível (MSi), fibra em detergente neutro indigestível (FDNi) e fibra em detergente ácido indigestível (FDAi)

Table 1 - Estimates of mean fecal concentration $\left(A_{O}-\%\right)$, maximal and minimal estimated fecal concentration (\%) and total oscillation amplitude for the markers chromium oxide, indigestible dry matter (DMi), indigestible neutral detergent fiber (NDFi) and indigestible acid detergent fiber (ADFi)

\begin{tabular}{|c|c|c|c|c|}
\hline \multirow[t]{2}{*}{$\begin{array}{l}\text { Indicador } \\
\text { Marker }\end{array}$} & \multirow[b]{2}{*}{$\mathrm{A}_{0}^{2}$} & \multicolumn{2}{|c|}{$\begin{array}{l}\text { Concentração estimada } \\
\text { Estimated concentration }\end{array}$} & \multirow[t]{2}{*}{$\begin{array}{c}\text { Amplitude de oscilação }(\%)^{3} \\
\text { Oscillation amplitude (\%) }\end{array}$} \\
\hline & & $\begin{array}{l}\text { Mínima } \\
\text { Minimum }\end{array}$ & $\begin{array}{r}\text { Máxima } \\
\text { Maximum } \\
\end{array}$ & \\
\hline $\begin{array}{l}\text { Óxido crômico } \\
\text { Chromium oxide }\end{array}$ & $0,210 \pm 0,00$ & 0,2046 & 0,2145 & 4,71 \\
\hline $\operatorname{MSi}(D M i)$ & $72,14 \pm 0,23$ & 71,47 & 72,79 & 1,83 \\
\hline FDNi (NDFi) & $54,90 \pm 0,20$ & 54,08 & 55,41 & 2,42 \\
\hline FDAi (ADFi) & $35,53 \pm 0,14$ & 35,20 & 35,82 & 1,74 \\
\hline
\end{tabular}

${ }^{1}$ Concentrações expressas como cromo, $\mathrm{Cr}^{3+}$ (Concentration expressed as chromium).

2 Estimativa \pm erro-padrão assintótico (Estimate \pm assimptotic standard error).

3 Amplitude de oscilação $=\left((\right.$ Máxima-Mínima $\left.) / A_{0}\right) \times 100\left(\right.$ Oscillation amplitude $=($ maximum - minimum $\left.\left.) / A_{0}\right) \times 100\right)$.

Tabela 2 - Estimativas, desvios-padrão assintóticos (DPA) e limites do intervalo de confiança assintóticos com 95\% de probabilidade $\left(\mathrm{ICA}_{95 \%}\right)$ para os períodos fundamentais dos ciclos de oscilação de concentração $(c$ - rad/h) e período fundamental do ciclo de oscilação $\left(t_{c}\right.$, hora) para os indicadores óxido crômico, matéria seca indigestível (MSi), fibra em detergente neutro indigestível (FDNi) e fibra em detergente ácido indigestível (FDAi)

Table 2 - Estimates, assimptotic standard deviation (ASD) and limits of assymptotic confidence intervals with 95\% probability $\left(A C l_{95 \%}\right)$ for fundamental periods of oscillation cycles of concentration ( $C-$ rad/h) and fundamental periods of the oscilation cycles (FP- $h$ ) for the markers chromium oxide, indigestible dry matter (DMi) indigestible neutral detergent fiber (NDFi) and indigestible acid detergent fiber (ADFi)

\begin{tabular}{|c|c|c|c|c|c|}
\hline \multirow[t]{2}{*}{$\begin{array}{l}\text { Indicador } \\
\text { Marker }\end{array}$} & \multirow[t]{2}{*}{ c } & \multirow[t]{2}{*}{$\begin{array}{l}\text { DPA } \\
A S D\end{array}$} & \multicolumn{2}{|c|}{$\begin{array}{l}\text { Limite } \mathrm{ICA}_{95 \%} \\
\text { Limit } \mathrm{ACI}_{95 \%}\end{array}$} & \multirow[t]{2}{*}{$t_{c}$} \\
\hline & & & $\begin{array}{l}\text { Inferior } \\
\text { Inferior }\end{array}$ & $\begin{array}{l}\text { Superior } \\
\text { Superior }\end{array}$ & \\
\hline Óxido crômico (Chromium oxide) & 0,9652 & 0,0874 & 0,7936 & 1,1369 & 3,25 \\
\hline MSi (DMi) & 0,9046 & 0,0489 & 0,8086 & 1,0006 & 3,47 \\
\hline FDNi (NDFi) & 0,9086 & 0,0476 & 0,8150 & 1,0021 & 3,46 \\
\hline
\end{tabular}

As estimativas do parâmetro $c$ obtidas neste trabalho foram significativamente diferentes de zero $(\mathrm{P}<0,05)$ para os indicadores $\mathrm{Cr}_{2} \mathrm{O}_{3}(0,9652 \pm 0,087 \mathrm{rad} / \mathrm{hora}), \mathrm{MSi}(0,9046 \pm 0,049$ $\mathrm{rad} /$ hora $), \operatorname{FDNi}(0,9086 \pm 0,048 \mathrm{rad} / \mathrm{hora})$ e FDAi $(0,840 \pm$ $0,045 \mathrm{rad} /$ hora), comprovando excreção fecal cíclica.

Este resultado indica, de forma preliminar, que períodos múltiplos de 3 horas poderiam ser empregados para representar adequadamente a concentração fecal média destes indicadores. No entanto, como os pontos devem ser amostrados antes e após as inflexões do perfil (Figura 1), o espaço de tempo entre duas coletas consecutivas deverá ser de $3,9,15$ ou 21 horas.

Os valores obtidos diferiram do encontrado por Hopper et al. (1978), cuja estimativa para o valor de $c$ não diferiu significativamente de $0,1309 \mathrm{rad} / \mathrm{hora}$, o que corresponde a uma duração para o período fundamental de 24 horas para o $\mathrm{Cr}_{2} \mathrm{O}_{3}$. Esses autores trabalharam com vacas em sistema de pastejo e, com base nesse valor, afirmaram que as coletas de fezes devem acompanhar os horários de fornecimento do indicador e propuseram coletas às $8 \mathrm{~h}$ e $17 \mathrm{~h}$.
Quando consideradas as concentrações esperadas às $7 \mathrm{~h}$ e às $17 \mathrm{~h}$ e calculadas suas médias aritméticas para estimar as concentrações médias diárias dos indicadores nas fezes, essas médias foram divididas pelas respectivas estimativas $\hat{\mathrm{A}}_{0}$ obtidas para os mesmos indicadores e foram obtidos os seguintes quocientes: 0,$9979 ; 1,0019 ; 1,0011$; 0,9986 , o que produziu média geral de 0,9999 , praticamente $100 \%$ de representatividade para as médias estimadas nestes horários em relação aos valores. Às $8 \mathrm{~h}$ e às $17 \mathrm{~h}$, esta média geral foi de 1,0006 ; às $6 \mathrm{~h}$ e às $17 \mathrm{~h}$, de 1,0010 ; e às $6 \mathrm{~h}$ e às $18 \mathrm{~h}$, igual a 0,9975 . Hopper et al. (1978) obtiveram coeficiente de regressão equivalente ao aplicarem um modelo de regressão linear sem intercepto. Esses autores concluíram que o coeficiente de regressão não desviou da unidade para amostras colhidas às $8 \mathrm{~h}$ e às $17 \mathrm{~h}$, isto é, $100 \%$ de representatividade em relação ao teor médio de cromo nas fezes, obtido em ensaio de digestão com vacas leiteiras em pastejo.

Os valores obtidos para a recuperação fecal dos indicadores em estudo (Tabela 3 ) permitem concluir pela melhor 
recuperação do $\mathrm{Cr}_{2} \mathrm{O}_{3}(96,92 \%)$, que não diferiu de $100 \%$, o que está de acordo com os valores obtidos em 90 estudos descritos na literatura, que apontam recuperação de $100 \%$ para o $\mathrm{Cr}_{2} \mathrm{O}_{3}$ como indicador externo (Titgemeyer, 1997).

Os resultados obtidos neste experimento para a recuperação fecal dos indicadores internos $(120,35 ; 120,80$ e $123,19 \%$, respectivamente, para MSi, FDNi e FDAi) apontam para superestimação desta recuperação, levando à subestimação da produção fecal se empregados esses indicadores. Zeoula et al. (2002), estudando a recuperação fecal dos indicadores CIA, CIDA, FDAi e FDNi, observaram recuperação média do indicador FDNi de 101,61\%, o que não diferiu de $100 \%$, portanto, foi semelhante à obtida pela coleta total. No entanto, a recuperação da FDAi foi de $89,76 \%$ e diferiu de $100 \%$, superestimando conseqüentemente a produção fecal. Piaggio et al. (1991) também não obtiveram bons resultados quando utilizaram a FDAi como indicador interno. Os valores de recuperação variaram entre 92,90 a 104,97\%, o que foi atribuído a problemas na filtragem durante as análises ou a variações associadas à digestão in situ, incluindo o tamanho do poro dos sacos de náilon utilizados por onde ocorrem possíveis perdas de material.

Alves et al. (2001), por sua vez, obtiveram bons resultados com a utilização da FDAi como indicador interno, em comparação à coleta total de fezes, e não notaram diferença significativa entre os métodos para a digestibilidade dos componentes avaliados. Freitas et al. (2002) não obtiveram resultados eficientes com o uso da FDNi e observaram que

Tabela 3 - Médias e limites do intervalo de confiança com 95\% de probabilidade para a recuperação fecal (\%) dos indicadores óxido crômico, matéria seca indigestível (MSi), fibra em detergente neutro indigestível (FDNi) e fibra em detergente ácido indigestível (FDAi)

Table 3 - Means and limits of confidence interval with 95\% of probability for fecal recovery (\%) of the markers chromium oxide, indigestible dry matter (DMi), indigestible neutral detergent fiber (NDFi) and indigestible acid detergent fiber (ADFi)

\begin{tabular}{|c|c|c|c|c|}
\hline \multirow[t]{2}{*}{$\begin{array}{l}\text { Indicador } \\
\text { Marker }\end{array}$} & \multirow[t]{2}{*}{$\begin{array}{l}\text { Média } \\
\text { Mean }\end{array}$} & \multirow[t]{2}{*}{$\mathrm{CV}(\%)^{1}$} & \multicolumn{2}{|c|}{$\begin{array}{c}\text { Limites } \mathrm{IC}_{95 \%} \\
\mathrm{CI}_{95 \%} \text { limits }\end{array}$} \\
\hline & & & $\begin{array}{l}\text { Inferior } \\
\text { Inferior }\end{array}$ & $\begin{array}{l}\text { Superior } \\
\text { Superior }\end{array}$ \\
\hline $\begin{array}{l}\text { Óxido crômico } \\
\text { Chromium oxide }\end{array}$ & 96,92 & 5,3 & 93,84 & 100,00 \\
\hline $\begin{array}{l}\mathrm{MSi} \\
\mathrm{DMi}\end{array}$ & 120,35 & 7,5 & 114,99 & 125,71 \\
\hline $\begin{array}{l}\mathrm{FDNi} \\
\mathrm{NDFi}\end{array}$ & 120,80 & 9,2 & 114,19 & 127,41 \\
\hline $\begin{array}{l}\text { FDAi } \\
A D F i\end{array}$ & 123,19 & 9,7 & 116,07 & 130,31 \\
\hline
\end{tabular}

\footnotetext{
${ }^{1}$ Variação ajustada para os efeitos de animal e período experimental
}

1 Variation adjusted for the effects of animal and experimental period. as estimativas de produção fecal obtidas com o uso da FDNi in vitro e FDNi in situ foram sub e superestimadas, respectivamente.

A variação na recuperação da FDNi pode ser causada por variações no tamanho das partículas dos substratos fermentados (Lippke et al., 1986), pela composição da fibra, no período de tempo e no modo de incubação (in vitro ou in situ) (Zeoula et al., 2002). Esses fatores de variação devem ser considerados quando utilizada FDNi como indicador interno.

De acordo com Huhtanen et al. (1994), a recuperação fecal de FDNi e FDAi foi mais acuradamente predita após 288 horas de incubação ruminal quando obtida pelo procedimento da incubação in vitro, portanto, a porosidade dos sacos de náilon se relacionou inversamente à acuidade das estimativas, que foi maior em $6 \mu \mathrm{m}$ que em $41 \mu \mathrm{m}$, comprovando influência do perfil das partículas empregadas.

Os problemas relacionados à utilização de resíduos indigestíveis da fibra parecem tender a um direcionamento comum, ou seja, as falhas na recuperação dos indicadores são reflexos diretos de falhas no processo de isolamento destes indicadores. Assim, estudos de validação e padronização dos métodos de avaliação são essenciais para se alcançar acuidade em diferentes situações e dietas.

\section{Conclusões}

A concentração média fecal dos indicadores estudados pode ser obtida por meio de duas coletas diárias de fezes, com intervalo de coletas consecutivas de $3,9,15$ ou 21 horas.

$\mathrm{O} \mathrm{Cr}_{2} \mathrm{O}_{3}$ apresenta a melhor recuperação fecal, que não difere de $100 \%$. As recuperações superestimadas dos indicadores internos podem estar relacionadas aos procedimentos para suas determinações.

\section{Literatura Citada}

ALVES, G.R.; FONTES, C.A.A.; ERBESDOBLER, E.D. et al. Influência do nível de consumo de pasto de capim-elefante sobre a composição da digesta e concentração do indicador $\mathrm{Cr}_{2} \mathrm{O}_{3}$ no trato gastrintestinal de bovinos. Revista Brasileira de Zootecnia, v.30, n.6, p.2110-2120, 2001.

BERCHIELLI, T.T.; ANDRADE, P.; FURLAN, C.L. Avaliação de indicadores internos em ensaios de digestibilidade. Revista Brasileira de Zootecnia, v.29, n.3, p.830-833, 2000.

BRISSON, G.J.; PIGDES, W.J.; SYLVESTRE, P.E. Effect of frequency of administration of chromic oxide on its fecal excretion pattern by grazing cattle. Canadian Journal Animal Science, v.37, n.1, p.90-94, 1957.

COCHRAN, R.C.; ADAMS, D.C.; WALLACE, J.D. et al. Predicting digestibility of different diets with internal markers: evaluation of four potential markers. Journal of Animal Science, v.63, n. 5 , p.1476-1483, 1986 
DETMANN, E.; PAULINO, M.F.; ZERVOUDAKIS, J.T. et al. Cromo e indicadores internos na determinação do consumo de novilhos mestiços, suplementados, a pasto. Revista Brasileira de Zootecnia, v.30, n.5, p.1600-1609, 2001

FAICHNEY, G.J. Digesta flow. In: FORBES, J.M.; FRANCE, J. (Eds.). Quantitative aspects of ruminant digestion and metabolim. Wallingford: CAB International, 1993. p.53-85.

FREITAS, D.; BERCHIELLI, T.T.; SILVEIRA, R.N. et al. Produção fecal e fluxo duodenal de matéria seca e matéria orgânica estimados por meio de indicadores. Revista Brasileira de Zootecnia, v.31, n.3, p.1521-1530, 2002 (supl.).

GOERING, H.K.; van SOEST, P.J. Forage fiber analyses. Washington: ARS-USDA, 1970. 20p. (Agriculture Handbook, 379).

HOPPER, J.T.; HOLLOWAY, J.W.; BUTtS JR., W.T. Animal variation in chromium sesquioxide excretion patterns of grazing cows. Journal of Animal Science, v.46, n.4, p.1098-1102, 1978 .

HUHTANEN, P.; KAUSTELL, K.; JAAKKOLA, S. The use of internal markers to predict digestibility and duodenal flow of nutrients in cattle given six different diets. Animal Feed Science and Technology, v.48, p.211-227, 1994.

KIMURA, F.T.; MILLER, V.L. Improved determination of chromic oxide in cal feed and feces. Journal of Agricultural Foodstuffs Chemistry, v.5, n.2, p.216, 1957.

LIPPKE, H.; ELLIS, W.C.; JACOBS, B.F. Recovery of indigestible fiber from feces of sheep and cattle on forage diets. Journal of. Dairy Science, v.69, n.2, p.403-412, 1986.

OLIVEIRA, R.F.M.; FONTES, C.A.A.; SILVA, J.F.C. et al. Estudo da recuperação feacal do $\mathrm{Cr}_{2} \mathrm{O}_{3}$ e dos indicadores internos, CIA, CIDA e lignina em períodos de coletas de dois a sete dias, em bovinos. Revista Brasileira de Zootecnia, v.20, n.5, p.522$531,1991$.
OWENS, F.N.; HANSON, C.F. External and internal markers for appraising site and extend of digestion in ruminants. Journal of Dairy Science, v.75, n.9, p.2605-2617, 1992.

PENNING, P.D.; JOHNSON, R.H. The use of internal markers to estimate herbage digestibility and intake. 2. Indigestible acid detergent fiber. Journal of Agricultural Science, v.100, n.1, p.133-138, 1983.

PIAGGIO, L.M.; PRATES, E.R.; PIRES, F.F. et al. Avaliação das cinzas insolúveis em ácido, fibra, em detergente ácido indigestível e lignina em detergente ácido indigestível como indicadores internos da digestibilidade. Revista da Sociedade Brasileira de Zootecnia, v.20, n.3, p.306-312, 1991.

STATISTICAL ANALYSIS SYSTEM - SAS. User's guide. Cary: 1993. 965p.

SILVA, D.J.; QUEIROZ, A.C. Análise de alimentos: métodos químicos e biológicos. 3.ed. Viçosa, MG: Editora UFV, 2002. $235 \mathrm{p}$.

TITGEMEYER, E.C. Design and interpretation of nutrient digestion studies. Journal of Animal Science, v.75, n.8, p.2235-2247, 1997.

Van SOEST, P.J. Nutrional ecology of the ruminant. 2.ed. Ithaca: Cornell University Press, 1994. 476p.

ZEOULA, L.M.; PRADO, I.N.; DIAN, P.H.M. et al. Recuperação fecal dos indicadores internos avaliados em ruminantes. Revista Brasileira de Zootecnia, v.31, n.4, p.1865-1874, 2002. 